\title{
TWO MIXED HADAMARD TYPE GENERALIZATIONS OF HEINZ INEQUALITY
}

\author{
TAKAYUKI FURUTA
}

(Communicated by John B. Conway)

Dedicated to Professor Zirô Takeda on his 65 th birthday with respect and affection

\begin{abstract}
We give two types of mixed Hadamard inequalities containing the terms $T,|T|$, and $\left|T^{*}\right|$, where $T$ is a bounded linear operator on a complex Hilbert space. As an immediate consequence of these results, we can easily show some extensions of the Hadamard inequality and also the Heinze inequality:

$$
|(T x, y)|^{2} \leq\left(|T|^{2 \alpha} x, x\right)\left(\left|T^{*}\right|^{2(1-\alpha)} y, y\right)
$$

for any $T$, any $x, y$ in $H$, and any real number $\alpha$ with $0 \leq \alpha \leq 1$. And the following conditions are equivalent in case $0<\alpha<1$ :

(1) the equality in $(*)$ holds;

(2) $|T|^{2 \alpha} x$ and $T^{*} y$ are linearly dependent;

(3) $T x$ and $\left|T^{*}\right|^{2(1-\alpha)} y$ are linearly dependent.

Results in this paper would remain valid for unbounded operators under slight modifications.
\end{abstract}

1. Introduction. An operator $T$ means a bounded linear operator on a complex Hilbert space $H$. We give two types of mixed Hadamard theorems containing the terms $T,|T|$, and $\left|T^{*}\right|$. These results are some extensions of Hadamard's theorem and the Heinz inequality

$$
|(T x, y)|^{2} \leq\left(|T|^{2 \alpha} x, x\right)\left(\left|T^{*}\right|^{2(1-\alpha)} y, y\right)
$$

for any $T$, any $x, y$ in $H$, and any real number $\alpha$ with $0 \leq \alpha \leq 1$. Also we scrutinize the cases when the equalities in these mixed Hadamard theorems hold. As an immediate consequence of this scrutiny, we can show that the equality in ( $\left.\mathrm{I}_{3}\right)$ holds if and only if $|T|^{2 \alpha} x$ and $T^{*} y$ are linearly dependent if and only if $T x$ and $\left|T^{*}\right|^{2(1-\alpha)} y$ are linearly dependent in case $\alpha$ with $0<\alpha<1$. Since $\left(\mathrm{I}_{3}\right)$ is equivalent to $\left(\mathrm{I}_{4}\right)$

$$
|(T x, y)| \leq\left\||T|^{\alpha} x\right\|\left\|\left|T^{*}\right|^{1-\alpha} y\right\|
$$

so that one might believe that the equality in $\left(\mathrm{I}_{3}\right)$ or $\left(\mathrm{I}_{4}\right)$ would hold if and only if $|T|^{2 \alpha} x$ and $\left|T^{*}\right|^{2(1-\alpha)} y$ are linearly dependent or $|T|^{\alpha} x$ and $\left|T^{*}\right|^{1-\alpha} y$ are linearly dependent. But here we can give a simple counter example to these mistakes. By this reason, the form of $\left(\mathrm{I}_{3}\right)$ is more convenient than $\left(\mathrm{I}_{4}\right)$ in order to remind us of the case when the equality in $\left(\mathrm{I}_{3}\right)$ or $\left(\mathrm{I}_{4}\right)$ holds. This paper extends the results obtained in [3 and 4].

Received by the editors January 26, 1987.

1980 Mathematics Subject Classification (1985 Revision). Primary 47A30; Secondary 47A99.

Key words and phrases. Heinz inequality, Hadamard inequality, mixed Schwarz inequality, polar decomposition.

(C) 1988 American Mathematical Society $0002-9939 / 88 \$ 1.00+\$ .25$ per page 


\section{Statement of the results.}

THEOREM 1 (MIXED HADAMARD TYPE 1). For any operator $T$ on $H$, any $x_{1}, x_{2}, \ldots, x_{n}$ in $H$, and any nonnegative number $\alpha$ and $\beta$ with $\alpha+\beta=1$, let $G_{n}$ be defined by

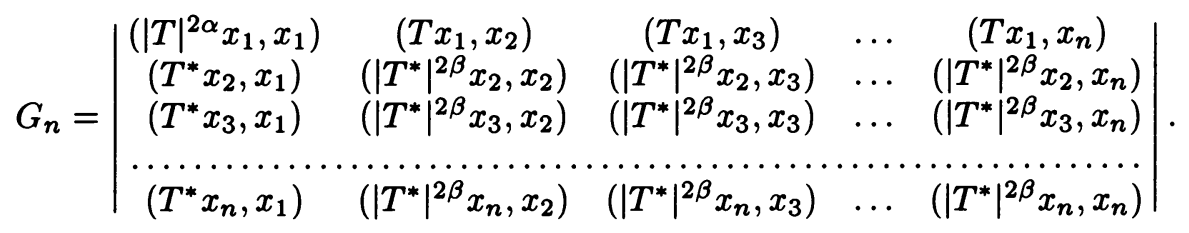

Then

$$
0 \leq G_{n} \leq\left\||T|^{\alpha} x_{1}\right\|^{2} \prod_{j=2}^{n}\left\|\left|T^{*}\right|^{\beta} x_{j}\right\|^{2} .
$$

Let $S_{1}(\alpha)$ and $S_{2}(\beta)$ be two systems of vectors defined by

$$
S_{1}(\alpha)=\left\{|T|^{2 \alpha} x_{1}, T^{*} x_{2}, T^{*} x_{3}, \ldots, T^{*} x_{n}\right\}
$$

and

$$
S_{2}(\beta)=\left\{T x_{1},\left|T^{*}\right|^{2 \beta} x_{2},\left|T^{*}\right|^{2 \beta} x_{3}, \ldots,\left|T^{*}\right|^{2 \beta} x_{n}\right\} .
$$

(1) $0<\alpha<1$. (i) $G_{n}=0$ iff $S_{1}(\alpha)$ is a system of linearly dependent vectors iff $S_{2}(\beta)$ is a system of linearly dependent vectors.

(ii) The following conditions (a) and (b) are equivalent:

(a) the right-hand side equality in $\left(\mathrm{I}_{1}\right)$ holds,

(b) the following $\left(\mathrm{b}_{1}\right)$ or $\left(\mathrm{b}_{2}\right)$ holds:

(b $\left.\mathrm{b}_{1}\right)\left(T x_{1}, x_{j}\right)=0$ for $j=2,3, \ldots, n$ and $\left(\left|T^{*}\right|^{2 \beta} x_{j}, x_{k}\right)=0$ for $j<k$ $(j=2,3, \ldots, n-1)$.

(b) $S_{1}(\alpha)$ contains the zero vector (equivalently, $S_{2}(\beta)$ contains the zero vector).

(2) $\alpha=1$. (i) $G_{n}=0$ iff $S_{2}(0)=\left\{T x_{1}, x_{2}, x_{3}, \ldots, x_{n}\right\}$ is a system of linearly dependent vectors.

(ii) The following conditions (a) and (b) are equivalent:

(a) the right-hand side equality in $\left(\mathrm{I}_{1}\right)$ holds,

(b) the following $\left(\mathrm{b}_{1}\right)$ or $\left(\mathrm{b}_{2}\right)$ holds:

(b $\left.\mathrm{b}_{1}\right)\left(T x_{1}, x_{j}\right)=0$ for $j=2,3, \ldots, n$ and $\left(x_{j}, x_{k}\right)=0$ for $j<k(j=2$, $3, \ldots, n-1)$.

$\left(\mathrm{b}_{2}\right) S_{2}(0)$ contains the zero vector.

(3) $\alpha=0$. (i) $G_{n}=0$ iff $S_{1}(0)=\left\{x_{1}, T^{*} x_{2}, T^{*} x_{3}, \ldots, T^{*} x_{n}\right\}$ is a system of linearly dependent vectors.

(ii) The following conditions (a) and (b) are equivalent:

(a) the right-hand side equality in $\left(\mathrm{I}_{1}\right)$ holds,

(b) the following $\left(\mathrm{b}_{1}\right)$ or $\left(\mathrm{b}_{2}\right)$ holds:

(b $\left.\mathrm{b}_{1}\right)\left(x_{1}, T^{*} x_{j}\right)=0$ for $j=2,3, \ldots, n$ and $\left(\left|T^{*}\right|^{2} x_{j}, x_{k}\right)=0$ for $j<k$ $(j=2,3, \ldots, n-1)$.

$\left(\mathrm{b}_{2}\right) S_{1}(0)$ contains the zero vector. 
THEOREM 2 (MIXED HADAMARD TYPE 2). For any operator $T$ on $H$, any $x_{1}, x_{2}, \ldots, x_{n}$ in $H$, and any nonnegative number $\alpha$ and $\beta$ with $\alpha+\beta=1$, let $G_{2 n}$ be defined by

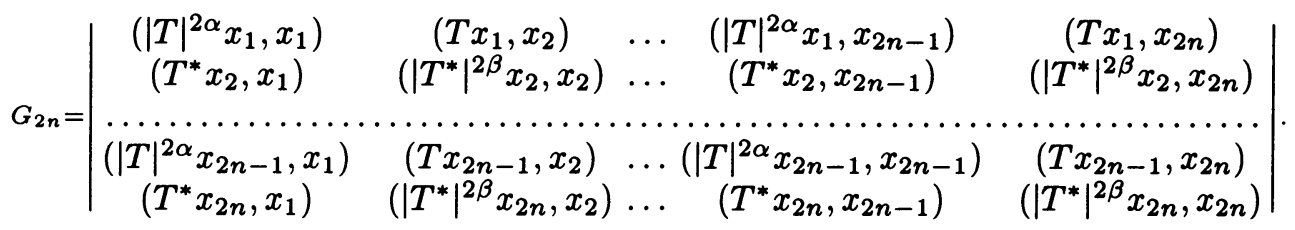

Then

$$
0 \leq G_{2 n} \leq \prod_{j=1}^{n}\left\||T|^{\alpha} x_{2 j-1}\right\|^{2}\left\|\left|T^{*}\right|^{\beta} x_{2 j}\right\|^{2} .
$$

Let $S_{1}(\alpha)$ and $S_{2}(\beta)$ be two systems of vectors defined by

$$
S_{1}(\alpha)=\left\{|T|^{2 \alpha} x_{1}, T^{*} x_{2},|T|^{2 \alpha} x_{3}, T^{*} x_{4}, \ldots,|T|^{2 \alpha} x_{2 n-1}, T^{*} x_{2 n}\right\}
$$

and

$$
S_{2}(\beta)=\left\{T x_{1},\left|T^{*}\right|^{2 \beta} x_{2}, T x_{3},\left|T^{*}\right|^{2 \beta} x_{4}, \ldots, T x_{2 n-1},\left|T^{*}\right|^{2 \beta} x_{2 n}\right\} .
$$

(1) $0<\alpha<1$. (i) $G_{2 n}=0$ iff $S_{1}(\alpha)$ is a system of linearly dependent vectors iff $S_{2}(\beta)$ is a system of linearly dependent vectors.

(ii) The following conditions (a) and (b) are equivalent:

(a) the right-hand side equality in $\left(\mathrm{I}_{2}\right)$ holds,

(b) the following $\left(\mathrm{b}_{1}\right)$ or $\left(\mathrm{b}_{2}\right)$ holds:

(b) $\left(\left|T^{*}\right|^{2 \beta} x_{2 j}, x_{2 k}\right)=0$ for $j \neq k,\left(|T|^{2 \alpha} x_{2 j-1}, x_{2 k-1}\right)=0$ for $j \neq k$ and $\left(T x_{2 j-1}, x_{2 k}\right)=0$ for $j, k=1,2, \ldots, n$.

$\left(\mathrm{b}_{2}\right) \quad 0 \in S_{1}(\alpha)$ (equivalently, $0 \in S_{2}(\beta)$ ).

(2) $\alpha=1$. (i) $G_{2 n}=0$ iff $S_{2}(0)=\left\{T x_{1}, x_{2}, T x_{3}, x_{4}, \ldots, T x_{2 n-1}, x_{2 n}\right\}$ is a system of linearly dependent vectors.

(ii) The following conditions (a) and (b) are equivalent:

(a) the right-hand side equality in $\left(\mathrm{I}_{2}\right)$ holds,

(b) the following $\left(\mathrm{b}_{1}\right)$ or $\left(\mathrm{b}_{2}\right)$ holds:

$\left(\mathrm{b}_{1}\right)\left(x_{2 j}, x_{2 k}\right)=0$ for $j \neq k,\left(|T|^{2} x_{2 j-1}, x_{2 k-1}\right)=0$ for $j \neq k$ and $\left(T x_{2 j-1}, x_{2 k}\right)=0$ for $j, k=1,2, \ldots, n$.

(b2) $0 \in S_{2}(0)$.

(3) $\alpha=0$. (i) $G_{2 n}=0$ iff $S_{1}(0)=\left\{x_{1}, T^{*} x_{2}, x_{3}, T^{*} x_{4}, \ldots, x_{2 n-1}, T^{*} x_{2 n}\right\}$ is a system of linearly dependent vectors.

(ii) The following conditions (a) and (b) are equivalent:

(a) the right-hand side equality in $\left(\mathrm{I}_{2}\right)$ holds,

(b) the following $\left(\mathrm{b}_{1}\right)$ or $\left(\mathrm{b}_{2}\right)$ holds:

$\left(\mathrm{b}_{1}\right)\left(\left|T^{*}\right|^{2} x_{2 j}, x_{2 k}\right)=0$ for $j \neq k,\left(x_{2 j-1}, x_{2 k-1}\right)=0$ for $j \neq k$ and $\left(x_{2 j-1}, T^{*} x_{2 k}\right)=0$ for $j, k=1,2, \ldots, n$.

$\left(\mathrm{b}_{2}\right) \quad 0 \in S_{1}(0)$.

COROLlaRY 1. For any operator $T$ on $H$,

$$
|(T x, y)|^{2} \leq\left(|T|^{2 \alpha} x, x\right)\left(\left|T^{*}\right|^{2(1-\alpha)} y, y\right)
$$

holds for any $x, y$ in $H$ and any real number $\alpha$ with $0 \leq \alpha \leq 1$. 
(1) $0<\alpha<1$. The equality in $\left(\mathrm{I}_{3}\right)$ holds iff $|T|^{2 \alpha} x$ and $T^{*} y$ are linearly dependent iff $T x$ and $\left|T^{*}\right|^{2(1-\alpha)} y$ are linearly dependent.

(2) $\alpha=1$. The equality in $\left(\mathrm{I}_{3}\right)$ holds iff $T x$ and $y$ are linearly dependent.

(3) $\alpha=0$. The equality in $\left(\mathrm{I}_{3}\right)$ holds iff $x$ and $T^{*} y$ are linearly dependent.

REMARK 1. $\left(\mathrm{I}_{3}\right)$ is obtained in $[\mathbf{1}, \mathbf{8}, \mathbf{9}]$ without the proof of the case when the equality in $\left(\mathrm{I}_{3}\right)$ holds. As $\left(\mathrm{I}_{3}\right)$ is equivalent to the following inequality $\left(\mathrm{I}_{4}\right)$ : $|(T x, y)| \leq\left\||T|^{\alpha} x\right\|\left\|\left|T^{*}\right|^{1-\alpha} y\right\|$, so that one might believe that the equality in $\left(\mathrm{I}_{3}\right)$ or $\left(\mathrm{I}_{4}\right)$ would hold iff $|T|^{2 \alpha} x$ and $\left|T^{*}\right|^{2(1-\alpha)} y$ are linearly dependent or $|T|^{\alpha} x$ and $\left|T^{*}\right|^{1-\alpha} y$ are linearly dependent. But here we can give a simple counterexample as follows. In case $\alpha=\frac{1}{2}$, let $T=\left(\begin{array}{ll}0 & 2 \\ 1 & 0\end{array}\right), x=\left(\begin{array}{l}1 \\ 1\end{array}\right)$ and $y=\left(\begin{array}{l}1 \\ 4\end{array}\right)$; then $\left|T^{*}\right| y=$ $\left(\begin{array}{l}2 \\ 4\end{array}\right)=2|T| x$, namely $|T| x$ and $\left|T^{*}\right| y$ are linearly dependent, but $|(T x, y)|^{2}=36 \neq$ $(|T| x, x)\left(\left|T^{*}\right| y, y\right)=54$. Also we can give an example such that the equality in $\left(\mathrm{I}_{3}\right)$ or $\left(\mathrm{I}_{4}\right)$ does not hold even if $|T| x$ and $\left|T^{*}\right|^{1-\alpha} y$ are linearly dependent. We would like to emphasize that the equality in $\left(\mathrm{I}_{3}\right)$ or $\left(\mathrm{I}_{4}\right)$ holds iff $|T|^{2 \alpha} x$ and $T^{*} y$ are linearly dependent iff $T x$ and $\left|T^{*}\right|^{2(1-\alpha)} y$ are linearly dependent in case $\alpha$ with $0<\alpha<1$. Corollary 1 is shown in [4].

REMARK 2. Put $\alpha=\frac{1}{2}$ in Corollary 1 ; then we have the useful inequality $|(T x, y)|^{2} \leq(|T| x, x)\left(\left|T^{*}\right| y, y\right)$, the so-called "mixed Schwarz inequality" [6] as an extension of $|(T x, y)|^{2} \leq(T x, x)(T y, y)$ for positive operator $T$. By Corollary 1, the equality in this mixed Schwarz inequality holds iff $|T| x$ and $T^{*} y$ are linearly dependent iff $T x$ and $\left|T^{*}\right| y$ are linearly dependent.

3. Proofs of the results. In order to show the results, we need the following

THEOREM A. For any $x_{1}, x_{2}, \ldots, x_{n} \in H$, let $G_{n}$ be the determinant of a square matrix of order $n$ defined by $G_{n}=\left|\left(\left(x_{j}, x_{k}\right)\right)\right|$. Then

$$
0 \leq G_{n} \leq\left\|x_{1}\right\|^{2}\left\|x_{2}\right\|^{2} \cdots\left\|x_{n}\right\|^{2} .
$$

On the left-hand side, equality holds if and only if $x_{1}, x_{2}, \ldots, x_{n}$ are linearly dependent. On the right-hand side, equality holds if and only if $x_{1}, x_{2}, \ldots, x_{n}$ are mutual orthogonal or $\left\{x_{1}, x_{2}, \ldots, x_{n}\right\}$ contains the zero vector.

The right-hand side inequality in Theorem A is Hadamard's theorem, and the left-hand side inequality in Theorem $\mathrm{A}$ is well known and can be considered as a generalization of Schwarz inequality. Many ingenious and elegant proofs of Hadamard's theorem have been given by many authors (for example, $[\mathbf{2}, \mathbf{5}, \mathbf{7}, \mathbf{1 1}]$ ).

LEMMA. Let $T=U|T|$ be the polar decomposition of $T$, where $U$ means the partial isometry and $|T|=\left(T^{*} T\right)^{1 / 2}$ with $N(U)=N(|T|)$, where $N(S)$ deriotes the kernel of an operator $S$. Then, for any positive number $p$,

$$
\left|T^{*}\right|^{p}=U|T|^{p} U^{*}
$$

PROOF. First of all, we state the following obvious but important relation $(*)$ : (*) $N\left(S^{q}\right)=N(S)$ for any positive operator $S$ and for any positive number $q$.

As $U^{*} U$ is the initial projection, then we have $U^{*} U|T|^{q}=|T|^{q}$ for any positive number $q$ because the relation $(*)$ for $|T|$ yields $\overline{R\left(|T|^{q}\right)}=\overline{R(|T|)}$. Then

$$
\left|T^{*}\right|^{2}=T T^{*}=U|T||T| U^{*}=U|T| U^{*} U|T| U^{*}=\left(U|T| U^{*}\right)^{2},
$$


so $\left|T^{*}\right|=U|T| U^{*}$ since $U|T| U^{*}$ is positive. By induction, we have $\left|T^{*}\right|^{n / m}=$ $U|T|^{n / m} U^{*}$ for any natural number $m$ and $n$ because $U^{*} U|T|^{q}=|T|^{q}$; then the continuity of $T$ yields $\left|T^{*}\right|^{p}=U|T|^{p} U^{*}$ by attending $n / m \rightarrow p$, so the proof of (i) is complete.

PROOF OF THEOREM 1. (1) $0<\alpha<1$. In Theorem A, we replace $x_{1}$ by $|T|^{\alpha} x_{1}$, and $x_{k}$ by $|T|^{\beta} U^{*} x_{k}$ for $k=2,3, \ldots, n$, and for any positive number $\alpha$ and $\beta$ with $\alpha+\beta=1$. Then, by the Lemma we have the following:

$$
\begin{gathered}
\left(|T|^{\alpha} x_{1},|T|^{\beta} U^{*} x_{k}\right)=\left(U|T| x_{1}, x_{k}\right)=\left(T x_{1}, x_{k}\right) \quad \text { for } k=2,3, \ldots, n, \\
\left(|T|^{\beta} U^{*} x_{j},|T|^{\beta} U^{*} x_{k}\right)=\left(U|T|^{2 \beta} U^{*} x_{j}, x_{k}\right)=\left(\left|T^{*}\right|^{2 \beta} x_{j}, x_{k}\right) \quad \text { for } j, k=2,3, \ldots, n .
\end{gathered}
$$

By Theorem A and the Lemma, we have

$$
\begin{aligned}
0 \leq G_{n} & \leq\left\||T|^{\alpha} x_{1}\right\|^{2}\left\||T|^{\beta} U^{*} x_{2}\right\|^{2}\left\||T|^{\beta} U^{*} x_{3}\right\|^{2} \cdots\left\||T|^{\beta} U^{*} x_{n}\right\|^{2} \\
& =\left\||T|^{\alpha} x_{1}\right\|^{2}\left\|\left|T^{*}\right|^{\beta} x_{2}\right\|^{2}\left\|\left|T^{*}\right|^{\beta} x_{3}\right\|^{2} \cdots\left\|\left.T^{*}\right|^{\beta} x_{n}\right\|^{2} .
\end{aligned}
$$

$G_{n}=0$ iff $|T|^{\alpha} x_{1},|T|^{\beta} U^{*} x_{2},|T|^{\beta} U^{*} x_{3}, \ldots,|T|^{\beta} U^{*} x_{n}$ are linearly dependent by Theorem A iff $|T|^{2 \alpha} x_{1},|T| U^{*} x_{2},|T| U^{*} x_{3}, \ldots,|T| U^{*} x_{n}$ are linearly dependent by the relation $(*)$ for $|T|$ iff $\left(D_{1}\right): S_{1}(\alpha)=\left\{|T|^{2 \alpha} x_{1}, T^{*} x_{2}, T^{*} x_{3}, \ldots, T^{*} x_{n}\right\}$ is a system of linearly dependent vectors.

On the other hand, $G_{n}=0$ iff $|T|^{\alpha} x_{1},|T|^{\beta} U^{*} x_{2},|T|^{\beta} U^{*} x_{3}, \ldots,|T|^{\beta} U^{*} x_{n}$ are linearly dependent iff $|T| x_{1},|T|^{2 \beta} U^{*} x_{2},|T|^{2 \beta} U^{*} x_{3}, \ldots,|T|^{2 \beta} U^{*} x_{n}$ are linearly dependent by the relation (*) for $|T|$, equivalently, $U|T| x_{1}, U|T|^{2 \beta} U^{*} x_{2}, U|T|^{2 \beta} U^{*} x_{3}, \ldots$, $U|T|^{2 \beta} U^{*} x_{n}$ are linearly dependent by $(*)$ for $|T|$ and $N(U)=N(|T|)$ iff $\left(\mathrm{D}_{2}\right)$ : $S_{2}(\beta)=\left\{T x_{1},\left|T^{*}\right|^{2 \beta} x_{2},\left|T^{*}\right|^{2 \beta} x_{3}, \ldots,\left|T^{*}\right|^{2 \beta} x_{n}\right\}$ is a system of linearly dependent vectors by (i) of the Lemma. Therefore, $\left(D_{1}\right)$ holds iff $\left(D_{2}\right)$ holds.

The proof of equality for the right-hand side of $\left(\mathrm{I}_{1}\right)$ follows by Theorem $\mathrm{A}$ and the argument stated above in the first half of the proof, so the proof of the case (1) is complete. The proofs of the case (2) $\alpha=1$ and (3) $\alpha=0$ are obvious, so we omit them. Hence we have finished the proof of Theorem 1 .

PROOF OF THEOREM 2. (1) $0<\alpha<1$. In Theorem $\mathrm{A}$, we replace $x_{2 k}$ by $|T|^{\beta} U^{*} x_{2 k}$ for $k=1,2, \ldots, n$, and $x_{2 k-1}$ by $|T|^{\alpha} x_{2 k-1}$ for $k=1,2, \ldots, n$, and for any positive number $\alpha$ and $\beta$ with $\alpha+\beta=1$. Then, by the Lemma we have the following:

$$
\begin{gathered}
\left(|T|^{\beta} U^{*} x_{2 j},|T|^{\beta} U^{*} x_{2 k}=\left(U|T|^{2 \beta} U^{*} x_{2 j}, x_{2 k}\right)=\left(\left|T^{*}\right|^{2 \beta} x_{2 j}, x_{2 k}\right)\right. \\
\left(|T|^{\alpha} x_{2 j-1},|T|^{\beta} U^{*} x_{2 k}\right)=\left(U|T| x_{2 j-1}, x_{2 k}\right)=\left(T x_{2 j-1}, x_{2 k}\right)
\end{gathered}
$$

for $j, k=1,2, \ldots, n$. By Theorem A and the Lemma, we have

$$
\begin{aligned}
0 & \leq G_{2 n} \leq\left\||T|^{\alpha} x_{1}\right\|^{2}\left\||T|^{\beta} U^{*} x_{2}\right\|^{2}\left\||T|^{\alpha} x_{3}\right\|^{2}\left\||T|^{\beta} U^{*} x_{4}\right\|^{2} \\
& \cdots\left\||T|^{\alpha} x_{2 n-1}\right\|^{2}\left\||T|^{\beta} U^{*} x_{2 n}\right\|^{2} \\
& =\left\||T|^{\alpha} x_{1}\right\|^{2}\left\|\left|T^{*}\right|^{\beta} x_{2}\right\|^{2}\left\||T|^{\alpha} x_{3}\right\|^{2}\left\|\left|T^{*}\right|^{\beta} x_{4}\right\|^{2} \cdots\left\||T|^{\alpha} x_{2 n-1}\right\|^{2}\left\|\left|T^{*}\right|^{\beta} x_{2 n}\right\|^{2} .
\end{aligned}
$$

The proofs of the left-hand side and right-hand side of the equality in $\left(\mathrm{I}_{2}\right)$ are given by the same way as in the proofs of Theorem 1 , so we omit them. The proofs of the case $\alpha=1$ and $\alpha=0$ are obvious, so we omit them. Hence we have finished the proof of Theorem 2.

PROOF OF COROLLARY 1. The proof follows by the left-hand side inequality of Theorem 1 or Theorem 2. 
Other generalizations of the Heinz inequality were obtained in [1 and 10]. Results in this paper would remain valid for unbounded operators under slight modifications.

The author would like to express his thanks to the referee for valuable commments.

\section{REFERENCES}

1. J. Dixmier, Sur une inegalité de E. Heinz, Math. Ann. 126 (1953), 75-78.

2. T. Furuta, An elementary proof of Hadamard's theorem, Mat. Vestnik 23 (1971), 267-269.

3. __ Mixed Hadamard's theorems, Proc. Amer. Math. Soc. 96 (1986), 217-220.

4. _ A simplified proof of Heinz inequality and scrutiny of its equality, Proc. Amer. Math. Soc. 97 (1986), 751-753.

5. F. G. Gantmacher, The theory of matrices, Vol. 1, Chelsea, New York, 1960.

6. P. Halmos, A Hilbert space problem book, 2nd ed., Springer-Verlag, Berlin and New York, 1982.

7. G. H. Hardy, J. E. Littlewood, and G. Polya, Inequalities, Cambridge Univ. Press, 1934.

8. E. Heinz, On an inequality for linear operators in a Hilbert space, Report on Operator Theory and Group Representations, no. 387, National Academy of Science, U.S.A., 1955, pp. 27-29.

9. T. Kato, Note on some inequalities for linear operators, Math. Ann. 125 (1952), 208-212.

10. __ A generalization of Heinz inequality, Proc. Japan Acad. 37 (1961), 305-308.

11. H. Widom, Lectures on integral equations, Van Nostrand, 1969.

Department of Mathematics, Faculty of Science, Hirosaki University, BunKYo-ChO 3, HiRosaki, AOMORI 036, JAPAN 